\title{
Bowel-Associated Dermatosis-Arthritis Syndrome in an Adolescent With Short Bowel Syndrome
}

\author{
Ester Pereira, MD, * Paula Estanqueiro, MD, * Susana Almeida, MD, * Ricardo Ferreira, MD, * \\ Oscar Tellechea, PhD, $†$ and Manuel Salgado, MD*
}

\begin{abstract}
Bowel-associated dermatosis-arthritis syndrome (BADAS) is a neutrophilic dermatosis, characterized by the occurrence of arthritis and skin lesions related to bowel disease with or without bowel bypass. We report an unusual case of BADAS in a 15-year-old white male with congenital aganglionosis of the colon and hypoganglionosis of the small intestine and multiple bowel surgeries in childhood complicated by short bowel syndrome. He presented with recurrent peripheral polyarthritis, tenosynovitis, and painful erythematous subcutaneous nodules located on the dorsolateral regions of the legs and on the dorsa of the feet. Histological examination disclosed a neutrophilic dermatosis confirming the diagnosis of BADAS.

Although an uncommon disease, especially at pediatric age, it is important to evoke the diagnosis of BADAS in children and adolescents with bowel disease, because treatment options and prognosis are distinct from other rheumatologic conditions.
\end{abstract}

Key Words: bowel-associated dermatosis-arthritis syndrome, neutrophilic dermatosis, arthritis, bowel disease, congenital aganglionosis, short bowel syndrome, adolescent

(J Clin Rheumatol 2014;20: 322-324)

$\mathrm{B}^{\mathrm{o}}$ owel-bypass syndrome is a well-recognized complication of jejunoileal bypass surgery for morbid obesity. It is characterized by the occurrence of arthritis and skin lesions days to many years after the surgery, affecting up to $20 \%$ of these patients. ${ }^{1-4}$ Bowel-bypass syndrome was first coined in 1979 by Dicken and Seehafer, ${ }^{1}$ who postulated that the pathogenesis was related to bacterial overgrowth in the blind loops of the bowel. The name bowel-associated dermatosis-arthritis syndrome (BADAS), proposed in 1983 by Jorizzo et al., ${ }^{5}$ was later adopted to include patients without bowel-bypass surgery, because the same syndrome was recognized in association with other surgical procedures and gastrointestinal diseases, such as inflammatory bowel disease. ${ }^{4-7}$

We present an unusual case report of BADAS, manifested by recurrent arthritis and painful subcutaneous nodules in a male adolescent with congenital aganglionosis of the colon and hypoganglionosis of the small intestine and multiple bowel surgeries in childhood complicated by short bowel syndrome.

\section{CASE REPORT}

On November 2008, a 15-year-old white male adolescent presented with a first episode of bilateral arthritis and tenosynovitis of the tibiotarsal joints. One month later, he had another episode of arthritis of the third and fourth metacarpophalangeal

From the *Hospital Pediátrico de Coimbra and $\dagger$ Department of Dermatology, Centro Hospitalar e Universitário de Coimbra, EPE, Coimbra, Portugal.

This study has no sources of funding.

The authors declare no conflict of interest.

Correspondence: Manuel Salgado, MD, Hospital Pediátrico de Coimbra, Av. Afonso Romão, Santo António dos Olivais, 3000-602 Coimbra. E-mail: mbsalgado27@gmail.com.

Copyright (C) 2014 by Lippincott Williams \& Wilkins

ISSN: 1076-1608

DOI: 10.1097/RHU.0000000000000137 joints of the right hand. He had morning stiffness and impaired function. He also complained of migrating polyarthralgias of the small joints of the hands and feet and Raynaud phenomenon (pale and cyanotic phases) in response to cold exposure. On physical examination, there was bilateral tibiotarsal swelling (including the Achilles tendons), erythema, and warmth, with limited joint excursion and pain on passive and active motion. Erythema overlaid the third and fourth metacarpophalangeal joints, without swelling but with pain on motion.

Medical history disclosed congenital aganglionosis of the colon and hypoganglionosis of the small intestine confirmed by intestinal biopsies. He had developed intestinal occlusion and undergone transverse colostomy at 4 days old, colectomy with ileorectal anastomosis at 19 months old, and subsequently 4 more bowel surgeries for intestinal occlusions, leading to short bowel syndrome. During childhood, multiple admissions were motivated by subocclusive episodes (last episode at 14 years old). On August 2008, he was diagnosed with nocturnal hypovision secondary to vitamin A deficiency, which improved after vitamin A supplementation.

He was initially managed with nonsteroidal anti-inflammatory drugs. Both episodes were self-limited, with 3 to 6 weeks' duration. Meanwhile, other liposoluble vitamin deficiencies were detected (vitamin D and vitamin E) and corrected. He also began parenteral nutrition every 2 weeks and oral antibiotherapy with metronidazole $30 \mathrm{mg} / \mathrm{kg}$ per day for 5 consecutive days, every month.

He became asymptomatic for about 1 year. On February 2010, at 16 years old, he restarted recurrent episodes of painful erythematous subcutaneous erythema nodosum-like nodules, lasting 1 to 4 weeks, with preferential location on the dorsolateral regions of the legs and on the dorsa of the feet (Fig. 1), occurring almost every month and accompanied by polyarthralgias or arthritis of the small joints of the hands and/or feet. Erythrocyte sedimentation rate varied between 26 and $42 \mathrm{~mm}$ (in the first hour) and C-reactive protein between 1.6 and $1.9 \mathrm{mg} / \mathrm{dL}$. Full blood cell count, renal function, electrolytes, protein immunoelectrophoresis, alanine and aspartate transaminases, uric acid, lactate dehydrogenase, serum angiotensin-converting enzyme, and $\mathrm{C} 4$ and $\mathrm{C} 3$ complement levels were within normal limits. Urinalysis was normal. Search for antinuclear antibodies and antineutrophil cytoplasmatic antibodies was negative. Nail-fold capillaroscopy yielded normal findings. Tuberculin skin test and interferon $\gamma$ release assay were negative. Human immunodeficiency virus infection, hepatitis $\mathrm{B}$, hepatitis $\mathrm{C}$, and recent group A $\beta$-hemolytic streptococcus infection were excluded. Upper gastrointestinal series showed marked dilatation of the small intestine and reduced peristalsis (Fig. 2). Skin biopsy (Fig. 3) revealed a dense neutrophilic infiltrate extending from the dermis to the subcutaneous fat, with perivascular and perieccrine topography in the dermis and diffuse lobular and septal infiltration of the hypodermis. Fibrinoid necrosis of small blood vessels and leukocytoclasis without erythrocyte extravasation were prominent in areas.

Adherence to the treatment was poor, including to the oral metronidazole, and he continued to have recurrent episodes of cutaneous and articular manifestations almost every 2 months. 

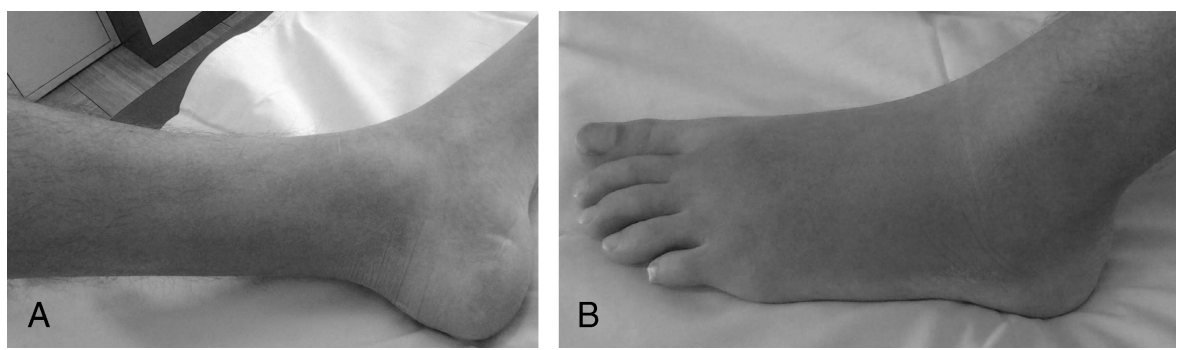

FIGURE 1. Painful erythematous subcutaneous erythema nodosum-like nodules on the dorsolateral regions of the legs (A) and on the dorsa of the feet (B).Color on-line figures available at http://www.jclinrheum.com

At 18 years old, he was transferred to the adult department of our hospital (September 2012). On October 2013, he had an episode of arthritis of the right knee and left elbow refractory to nonsteroidal anti-inflammatory drugs, which required intra-articular injection with triamcinolone hexacetonide. Treatment was changed to minocycline $100 \mathrm{mg} / \mathrm{d}$, sulfasalazine (Salazopyrin) $2500 \mathrm{mg} / \mathrm{d}$, and prednisolone $10 \mathrm{mg} / \mathrm{d}$. Disease control was achieved, and prednisolone dose was tapered to $5 \mathrm{mg} / \mathrm{d}$. On April 2014, he had another episode with articular and cutaneous manifestations that required a transient increase of the systemic corticosteroid dose. It was preceded by obstipation that occurred after taking oral flucloxacillin because of a soft tissue infection. Since then, he has been asymptomatic and actually (May 2014) continues to take minocycline $100 \mathrm{mg} / \mathrm{d}$, sulfasalazine $2500 \mathrm{mg} / \mathrm{d}$, and prednisolone $5 \mathrm{mg} / \mathrm{d}$.

\section{DISCUSSION}

Clinical features of recurrent polyarthritis, tenosynovitis, and painful erythematous subcutaneous nodules in an adolescent with short bowel syndrome were consistent with the diagnosis of BADAS. Conditions in association with BADAS are indicated in the Table. Bowel-associated dermatosis-arthritis syndrome has been described in a few patients with inflammatory bowel disease $^{5-8}$ and sporadically in patients with other miscellaneous gastrointestinal diseases. ${ }^{9-11}$ It occurs infrequently after modern surgical abdominal procedures. ${ }^{7}$

The pathogenesis of BADAS is currently poorly understood. Bowel stasis-promoting bacterial overgrowth and bacterial translocation could cause abnormal activation of the immune response, together with vitamin and mineral deficiencies, in particular of vitamin D. ${ }^{4}$ Formation and deposition of circulating bacterial immune complexes in the skin and synovial membrane may elicit the cutaneous and articular manifestations of BADAS. ${ }^{4}$ The histological finding of cutaneous small vessel vasculitis, as shown in our patient, fits with this hypothesis.
We suggest that congenital hypoganglionosis of the small intestine and multiple bowel surgeries in childhood (including colectomy and ileorectal anastomosis) may contribute to bowel stasis and be associated to BADAS. On the other hand, the absence of colon and ileocecal valve could have facilitated bacterial contamination of the entire malfunctioning bowel, increasing the likelihood of bacterial overgrowth and immune dysfunction.

Bowel-associated dermatosis-arthritis syndrome is almost always described in adult patients ${ }^{5-7}$ probably because of its high incidence after jejunoileal bypass surgery. To our knowledge, this is the second case report of BADAS occurring at pediatric age, the other being an 11-year-old male adolescent with Crohn disease. ${ }^{8}$

Clinical manifestations of BADAS may be self-limiting or chronic and recurrent, consisting of a combination of arthralgia, nonerosive polyarthritis, myalgia, and skin lesions. ${ }^{5}$ A prodromal febrile influenza-like illness may occur in $10 \%$ to $20 \%$ of patients. As in our case report, joint symptoms are usually described as migratory and polyarticular, with preferential involvement of the peripheral joints and accompanying tenosynovitis, but with no long-term damage or deformity. ${ }^{5-7}$ Raynaud phenomenon may also occur. ${ }^{3}$ We did not observe the skin lesions of BADAS, often described as characteristic and that appear in crops as small erythematous macules on the extremities and upper part of the trunk, progressing into sterile vesiculopustules over a 24 - to 48 -hour period and healing without scarring over about 2 weeks. ${ }^{1,2}$ However, manifestations can be protean and include panniculitis, nodules, erythematous plaques, and erythema nodosum-like skin eruptions, ${ }^{2}$ similarly to our case report. Skin lesions may recur every 4 to 6 weeks. ${ }^{1-4}$

Bowel-associated dermatosis-arthritis syndrome is part of a group of related cutaneous disorders called neutrophilic dermatoses. $^{12}$ They are rare disorders, especially in children, sharing similar clinical appearances and associated conditions including inflammatory bowel diseases, connective tissue diseases, myeloproliferative disorders (with immunoglobulin A monoclonal gammopathy), and medications. ${ }^{12}$ In our case report, histological findings were compatible with the diagnosis of BADAS, by
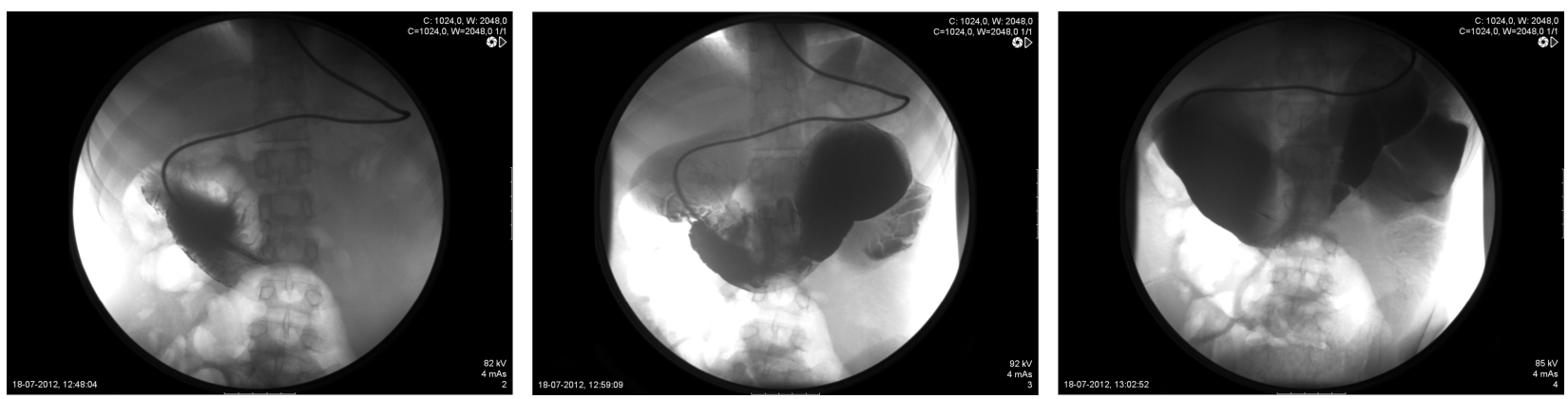

FIGURE 2. Upper gastrointestinal series. Marked dilatation and reduced peristalsis of the small intestine. 

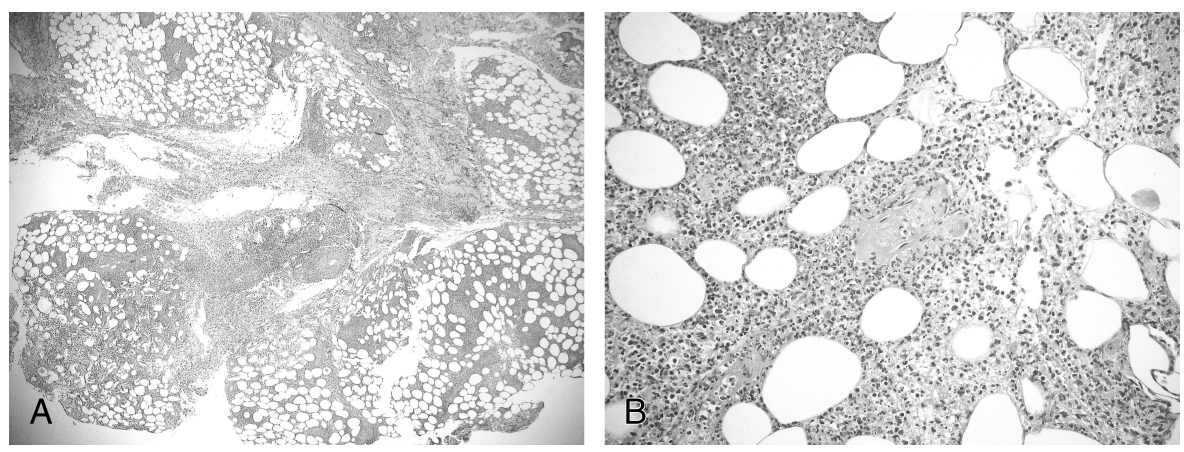

FIGURE 3. Histology of skin biopsy. Dense neutrophilic infiltrate extending from the dermis to the subcutaneous fat (A). Fibrinoid necrosis of a small blood vessel (B).Color on-line figures available at http://www.jclinrheum.com

demonstrating a dense dermal-hypodermal neutrophilic infiltrate. $^{2,7,12}$ Vasculitic changes with fibrinoid necrosis and leukocytoclasis, as well as panniculitis, are occasional findings described in the literature. ${ }^{12}$ On the other hand, histological findings also helped in distinguishing BADAS hypodermal neutrophilic infiltrates from erythema nodosum because in the latter no massive lobular infiltration is usually present.

In many cases, BADAS resolves spontaneously. ${ }^{7}$ When persistent or with particularly serious manifestations, treatment is directed to the correction of the bacterial overgrowth. ${ }^{1,4-7}$ Surgical reduction of the blind loops or restoration of the normal bowel anatomy has been curative. ${ }^{1,4}$ Long-term antibiotics such as metronidazole, erythromycin, and tetracyclines (including minocycline) have been used to decrease bacterial load in the affected bowel and suppress symptoms. ${ }^{5-7}$ Relief, however, may be only temporary, as observed in our case report. It may be useful to rotate antibiotics. ${ }^{4}$ As the group of the neutrophilic dermatoses, patients with BADAS may also benefit from the treatment with systemic corticosteroids, cyclosporine, and other drugs affecting the function of neutrophils, such as colchicine, dapsone, or Salazopyrin. ${ }^{46,13}$ In our case, improvement of the symptoms was obtained with minocycline, Salazopyrin, and prednisolone. Prednisolone dose should be discontinued when possible. Systemic corticosteroids may be helpful in a flare, but they are not adequate for longterm therapy. ${ }^{7}$

Although an uncommon disease, especially at the pediatric age, it is important to remember the diagnosis of BADAS in

TABLE. Gastrointestinal Conditions Described in Association With BADAS

Surgical procedures

Jejunoileal bypass $^{1}$
Jejunocolic bypass
Ileocolic bypass $^{1}$
Billroth II gastrectomy
Roux-en-Y jejunectomy $^{6}$
Ileoanal pouch anastomosis $^{14}$
Biliopancreatic diversion
${ }^{15}$
Laparoscopic gastric bypass $^{16}$
Inflammatory bowel disease $^{5-8}$
Gastric phytobezoar
Diverticulitis with sigmoid stenosis
Acute appendicitis
${ }^{11}$
Short bowel syndrome (congenital
aganglionosis of the colon and
hypoganglionosis of the small
intestine) (our case report)

Gastrointestinal diseases children and adolescents with chronic bowel disease presenting with arthritis and skin lesions, because treatment options and prognosis are distinct from other rheumatologic conditions.

\section{REFERENCES}

1. Dicken CH, Seehafer JR. Bowel bypass syndrome. Arch Dermatol. 1979; 115:837-839.

2. Kennedy $\mathrm{C}$. The spectrum of inflammatory skin disease following jejuno-ileal bypass for morbid obesity. Br J Dermatol. 1981;105:425-435.

3. Stein HB, Schlappner OL, Boyko W, et al. The intestinal bypass: arthritis-dermatitis syndrome. Arthritis Rheum. 1981;24:684-690.

4. Carubbi F, Ruscitti P, Pantano I, et al. Jejunoileal bypass as the main procedure in the onset of immune-related conditions: the model of BADAS. Expert Rev Clin Immunol. 2013;9:441-452.

5. Jorizzo JL, Apisarnthanarax P, Subrt P, et al. Bowel-bypass syndrome without bowel bypass: bowel-associated dermatosis-arthritis syndrome. Arch Intern Med. 1983;143:457-461.

6. Dicken $\mathrm{CH}$. Bowel-associated dermatosis-arthritis syndrome: bowel bypass syndrome without bowel bypass. J Am Acad Dermatol. 1986;14:792-796.

7. Thrash B, Patel M, Shah KR, et al. Cutaneous manifestations of gastrointestinal disease: part II. J Am Acad Dermatol. 2013;68:211.e1-211.e33.

8. Guerre-Schmidt AR, Pelletier F, Carbonnel F, et al. Dermatosis-arthritis syndrome associated with Crohn's disease in a teenager [in French]. Rev Med Interne. 2006;27:874-877.

9. Kemp DR, Gin D. Bowel-associated dermatosis-arthritis syndrome. Med J Aust. 1990;152:43-45.

10. Brouard MC, Chavaz P, Borradori L. Acute pustulosis of the legs in diverticulitis with sigmoid stenosis: an overlap between between bowel-associated dermatosis-arthritis syndrome and pustular pyoderma gangrenosum. J Eur Acad Dermatol Venereol. 2004;18:89-92.

11. Prpic-Massari L, Kastelan M, Brajac I, et al. Bowel-associated dermatosis-arthritis syndrome in a patient with appendicitis. $\mathrm{Med} \mathrm{Sci}$ Monit. 2007;13:CS97-CS100.

12. Berk DR, Bayliss SJ. Neutrophilic dermatoses in children. Pediatr Dermatol. 2008;25:509-519.

13. Cohen PR. Neutrophilic dermatoses: a review of current treatment options Am J Clin Dermatol. 2009;10:301-312.

14. Cox NH, Palmer JG. Bowel-associated dermatitis-arthritis syndrome associated with ileo-anal pouch anastomosis, and treatment with mycophenolate mofetil. Br J Dermatol. 2003;149:1296-1297.

15. Slater GH, Kerlin P, Georghiou PR, et al. Bowel-associated dermatosis-arthritis syndrome after biliopancreatic diversion. Obes Surg. 2004; 14:133-135.

16. Tu J, Chan JJ, Yu LL. Bowel bypass syndrome/bowel-associated dermatosis arthritis syndrome post laparoscopic gastric bypass surgery. Australas $J$ Dermatol. 2011:52:e5-e7. 